\title{
Comparison of Off-line Visual and Verbal Feedback Instructions for Keeping Tempo in Music
}

\author{
Hideyuki Tanaka ${ }^{1, *}$, Keita Ueda ${ }^{2}$ \\ ${ }^{1}$ Graduate School of Education, Hiroshima University, Higashi-hiroshima, Hiroshima 739-8524, Japan \\ ${ }^{2}$ Department of Education, Hiroshima University, Higashi-hiroshima, Hiroshima 739-8524, Japan
}

\section{ARTICLE INFO}

\section{Article History}

Received 18 May 2018

Accepted 14 November 2018

Key words

Rhythm in music

ability of keeping steady tempo

visual feedback

verbal feedback

\begin{abstract}
Ability of keeping the tempo is one of the important factors in playing music. In this paper, the authors carry out comparative experiments on the effects of off-line visual and verbal feedback instructions for informing the performance in trying to keep the tempo at $1 \mathrm{~Hz}$ by tapping the rhythm. The participants are divided into experienced and non-experienced groups. The results show that visual feedback may give too much information to experts of music and that experts can adjust the tempo by just using the verbal feedback.
\end{abstract}

(C) 2018 The Authors. Published by Atlantis Press SARL. This is an open access article under the CC BY-NC license (http://creativecommons.org/licenses/by-nc/4.0/).

\section{INTRODUCTION}

In music, rhythm is one of the important factors, and keeping the steady tempo is the basis for playing music. Rhythm is the component of music that punctuates time, carrying us from one beat to the next and subdivides into simple ration just like pitch [1].

In the classical method of teaching music, the teacher gives instructions and feedback on the performance of the student, commonly providing the student with feedback using imagery, for example, "sing as if through the top of your head" [2]. The effects of feedback instructions in music have been thus investigated by recording the process of teaching (e.g., Janice and Killian [3], Yarbrough et al. [4]). Comparison of the different feedback instructions in dance was carried out recently [5], but there are few comparison studies in music within last 3 years.

On-line visual feedback has been investigated for music instructions. It was reported by Sadakata et al. [6] that abstract shapes are useful for conveying on-line feedback information of timing and loudness; see also e.g., Hoppe et al. [2] for researches of on-line visual feedback for music instructions.

Off-line feedback has been used in the traditional education of music and it will be also used in future. It would be hence worth investigating, though on-line feedback is effective because of no time lag between the student's performance and teacher's feedback [2]. As far as the authors' knowledge, effects of off-line visual and verbal feedback instructions have not been compared and investigated in the literature. We therefore carry out experiments for comparing the effects of off-line visual and verbal feedback instructions in trying to keep the tempo constant at $1 \mathrm{~Hz}$.
Chen et al. [7] reported that the variability of synchronization with visual or verbal feedback depends on the frequency. Effects of practice on variability in an isochronous serial interval production task was studied by Madison et al. [8], but not focusing on comparing feedback instructions.

\section{EXPERIMENTAL SETUP AND FEEDBACK}

\subsection{Experimental Setup}

We show a schematic picture of the experimental setup in Figure 1. The participants beat the rhythm, holding a stick by their dominant hand and tapping the dram pad. The microphone captures the sound of the beat and sends the signals to the laptop PC of the windows OS. The PC analyzes the signals, obtains the time of the impacts by removing reverberation, and provides information for visual and verbal feedback. In an offline setup, visual or the verbal feedback is given by the display or by the language.

We assign the speed of the beat as 60 beats/min $(1 \mathrm{~Hz})$, and the participants try to keep the designated tempo in $2 \mathrm{~min}$. Before the experiments, we ask the participants to maintain the tempo as close to the designated one as possible. We carry out experiments of 10 trials for each participant and give visual or verbal feedback after each trial in an off-line setting.

\subsection{Feedback for Informing Performance}

Figure 2 shows a graph used for visually informing the periods between impacts. We inform participants how to read the graph, 


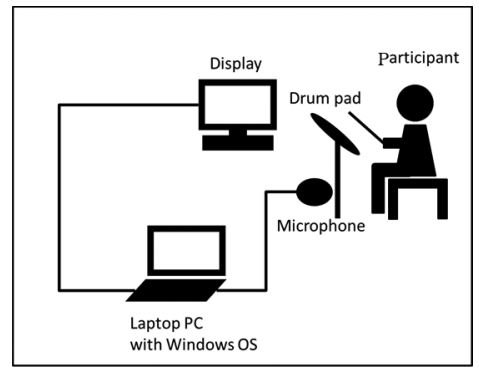

Figure $1 \mid$ A schematic picture of the experimental setup

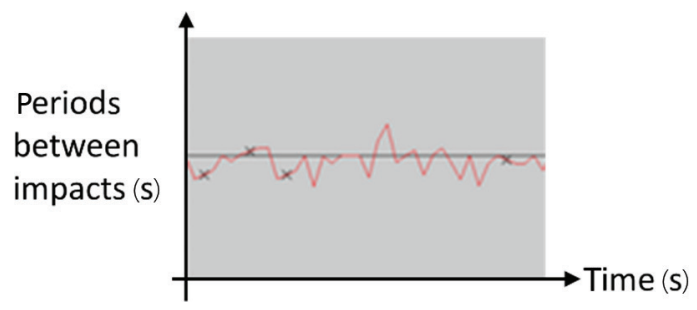

Figure 2 The graph showing the tempo and time. The horizontal and vertical axes respectively express time and the periods between impacts. The red line shows the result of the performance, where black crosses are estimated from interpolation because of missing data. The constant line is the designated period; if all the periods are closer to the designated one, it indicates that the participant taps the rhythm better

and they can hence visually understand how she/he tapped the rhythm in the trial.

We give verbal feedback based on numerical values calculated by the laptop computer. To this end, we define evaluation criteria for giving judges of the tendency. Dividing the time interval of the experiment into the first and second half, we analyze whether she/ he keeps the tempo and taps the rhythm in the designated speed.

For giving verbal feedback, we moreover calculate average tempos of the first and second half and compute the difference between them. The evaluation criteria are as follows: Let $y(k)$ be the $k$-th period, where $k=1,2,3, \ldots, N$. The number $N_{\mathrm{p}}$ of the first half data is given by $N_{\mathrm{p}}=N / 2$ or $N_{\mathrm{p}}=(N+1) / 2$ respectively for $N$ even or odd, and the number $N_{\mathrm{f}}$ of the second half is $N_{\mathrm{f}}=N-N_{\mathrm{p}}$. To judge whether the rhythm is kept steady, we define the following criteria respectively for the first and second half:

$$
\begin{aligned}
& H_{\mathrm{p}}=\max _{k=1,2, \cdots, N_{\mathrm{p}}}(|e(k)|)-\min _{k=1,2, \cdots, N_{\mathrm{p}}}(|e(k)|), \\
& H_{\mathrm{f}}=\max _{k=N_{\mathrm{p}}+1, \cdots, N}(|e(k)|)-\min _{k=N_{\mathrm{p}}+1, \cdots, N}(|e(k)|),
\end{aligned}
$$

where $e(k)$ is the error between the designated period $r$ and the $k$-th period $y(k)$ :

$$
e(k)=r-y(k)
$$

The sums of squares of the errors for the first and second half are respectively defined as follows:

$$
P_{\mathrm{p}}=\sum_{k=1}^{N_{\mathrm{p}}}(e(k))^{2}, \quad P_{\mathrm{f}}=\sum_{k=N_{\mathrm{p}}+1}^{N}(e(k))^{2} .
$$

The average periods of the first and second half are respectively given by

$$
M_{\mathrm{p}}=\frac{1}{N_{\mathrm{p}}} \sum_{k=1}^{N_{\mathrm{p}}} y(k), \quad M_{\mathrm{f}}=\frac{1}{N_{\mathrm{f}}} \sum_{k=N_{\mathrm{p}}+1}^{N} y(k),
$$

and the difference between them by $M_{c}=\left|M_{p}-M_{f}\right|$ and $M_{d}=M_{p}-$ $M_{f}$. We also compute the mean $\mu$ and the variance $\sigma^{2}$ of $y(k)$.

We give verbal feedback to the participants for the first and second half as follows:

- Good in the first (or second) half: Tapping the rhythm in the designated tempo, if $H_{p}<0.15$ and $P_{p}<0.1$ hold (or if $H_{f}<0.15$ and $P_{f}<0.1$ hold).

- Fairly good in the first (or second) half: Tapping the steady rhythm in the designated tempo but not keeping it in the designated tempo, if $H_{p}<0.15$ only holds (or if $H_{f}<0.15$ only holds).

- Not good in the first (or second) half: Tapping the rhythm not in the designated tempo, if $H_{p}>0.15$ holds (or if $H_{f}>0.15$ holds).

Concerning the average speeds for the first and second half, we moreover give the following feedback:

- Slow in the first (or second) half: The beat is slower than the designated tempo, if $M_{p}>1.05$ holds (or if $M_{f}>1.05$ holds).

- Fast in the first (or second) half: The beat is faster than the designated tempo, if $M_{p}<0.95$ holds (or if $M_{f}<0.95$ holds).

Comparing the speeds of the first and second half, we further give the following feedback:

- Getting slower: The tempo of the second half is slower than the first one, if $M_{d}<0$ and $M_{c}>0.05$.

- Getting faster: The tempo of the second half is slower than the first one, if $M_{d}>0$ and $M_{c}>0.05$.

\subsection{Experiments}

The number of participants is 45 , including 27 persons who have enough experiences of playing music and 18 persons who have not. We divide them into experienced and non-experienced groups, by asking them whether they have experiences of playing the instruments besides learning at class rooms in elementary, junior high or high schools. We also divide them into three groups of giving visual feedback (nine from 27 experienced and six from 18 non-experienced, verbal feedback (nine from 27 experienced, six from non-experienced), and no feedback (nine from 27 experienced, six from 18 non-experienced).

The participants first listen to the signal of 60 beats/min for the reference, where they can choose just listening to the signal or beating the drum pad according to the signal. They then try to maintain the tempo in $120 \mathrm{~s}$. After the trial, we give visual or verbal feedback to the participants in the groups of under feedback. We carry out 10 trials and give them 5 min of rests after the 4 th and 7 th trials, by taking physical and mental fatigues of participants into account. 


\section{RESULTS OF EXPERIMENTS}

We first compare the results of experienced and non-experienced participants of tapping the rhythm, focusing on the evaluation values $P_{p}$ and $P_{f}$. As we expected, the errors $e(k)$ of experienced participants is smaller than those of non-experienced participants in all the groups of visual, verbal and no feedback. We thus do not show the figure here.

We next compare the effects of visual, verbal, and no feedback. Figures 3 and 4 show the sums of squares $P_{p}$ and $P_{f}$ of the error $e(k)$ respectively for experienced and non-experienced participants. Both of the figures show that the participants under visual or verbal feedback (F.B.) can maintain the error smaller than those under no feedback. Figure 3 indicates that verbal feedback is more effective than visual feedback for the experienced participants.

We further compare the effects of visual, verbal, and no feedback by using the variance $\sigma^{2}$. Figures 5 and 6 show $\sigma^{2}$ of experts and non-experts, respectively. We see from Figures 3 and 5 that experienced participants can suppress the sums of squares of the error $e(k)$ by using visual feedback, but not the variance $\sigma^{2}$. The result implies that they can recognize the error in average (in the sense of the sum of squares) and that they can accelerate or decelerate by looking at the graph, if they beat slow or fast respectively. However, by looking at the graph, they cannot properly control the variance of periods. On the other hand, verbal feedback, often used for instruction in music plays, gives better results than the visual one, as shown in Figures 3 and 5.

Regarding the variance, we have also obtained information through questionnaire, by asking how they thought of feedback between trials; they answered: "To achieve better performance, I don't worry about the details.", "I tapped the rhythm without thinking too much.", and "I just cared the intervals of the reference signals between trials." It seems that several experienced

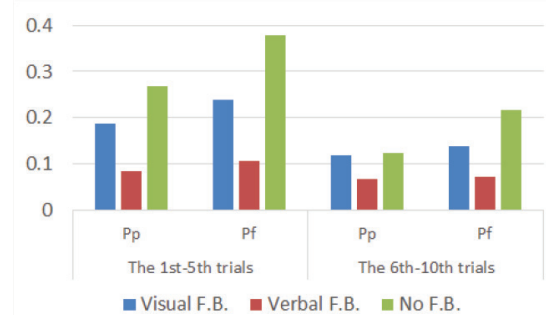

Figure 3 The sums of squares $P_{\mathrm{p}}$ and $P_{\mathrm{f}}$ of the errors for experienced participants

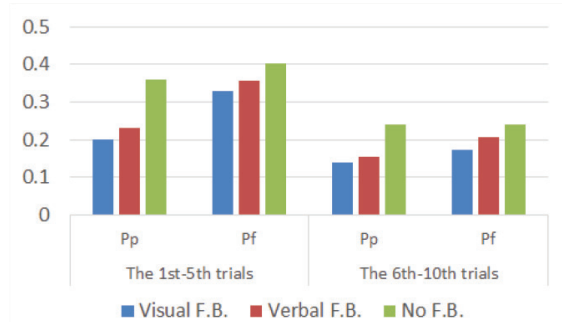

Figure 4 The sums of squares $P_{\mathrm{p}}$ and $P_{\mathrm{f}}$ of the errors for non-experienced participants

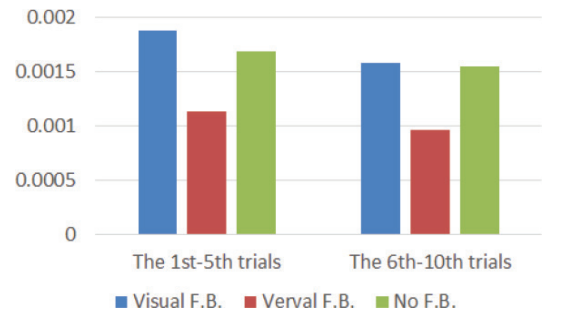

Figure 5 Variances $\sigma^{2}$ of experienced participants

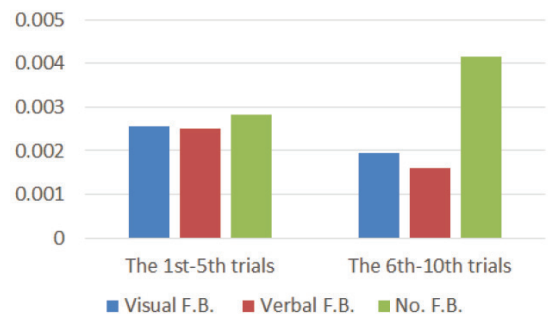

Figure 6 Variances $\sigma^{2}$ of non-experienced participants

participants thus considered that tapping the rhythm without being influenced too much by the instruction of the graph leads to better performances.

Summarizing the above results, visual feedback by the graph possibly provided too much information for the experienced participants to maintain the variance of the rhythm, whereas verbal feedback did appropriate information to them. Experienced participants would thus confused by the difference between their own feeling of rhythm and feedback indicated by the graph, since bodily process, rhythm, and physical motion is the basis of musical expressivity (Émile Jaques-Dalcroze [9]), and since bodily process and physical motion depend on their own bodies.

\section{CONCLUSION}

We have carried out experiments of training for keeping the steady tempo in music. We compared the effects of visual and verbal feedback for informing and improving their performance. The results indicated that visual feedback by the graph seems to give too much information to the experienced participants, and it may disturb the expert participants in maintaining the steady tempo, because they have their own feeling of rhythms. It is hence desirable for the teacher to send acceptable feedback to the student from the viewpoint of music education. Results may be improved by visual feedback, if we simplify the graph.

\section{REFERENCES}

[1] J. Martineau, The Elements of Music: Melody, Rhythm, and Harmony, Bloomsbury USA, New York, 2008.

[2] D. Hoppe, M. Sadakata, P. Desain, Development of real-time visual feedback assistance in singing training: a review, J. Comput. Assist. Learning 22 (2006), 308-316. 
[3] J.K.N. Killian, Effect of instructions and feed-back on music teaching skills, J. Music Ther. 18 (1981), 166-180.

[4] C. Yarbrough, J. Wapnick, R. Kelly, Effect of videotape feedback techniques on performance, verbalization, and attitude of beginning conductors, J. Res. Music Educ. 27 (1979), 103-112.

[5] L.H. Hsia, I. Huang, G.J. Hwang, Effects of different online peer-feedback approaches on students' performance skills, motivation and self-efficacy in a dance course, Comput. Educ. 96 (2016), 55-71.

[6] M. Sadakata, D. Hoppe, A. Brandmeyer, R. Timmers, P. Desain, Real-time visual feedback for learning to perform short rhythms

\section{Authors Introduction}

\section{Mr. Hideyuki Tanaka}

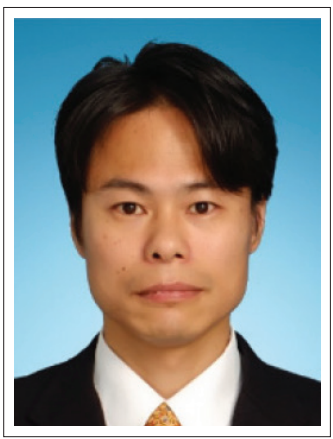

with expressive variations in timing and loudness, J. New Music Res. 37 (2008), 207-220.

[7] Y. Chen, B.H. Repp, A.D. Patel, Spectral decomposition of variability in synchronization and continuation tapping: comparisons between auditory and visual pacing and feedback conditions, Hum. Mov. Sci. 21 (2002), 515-532.

[8] G. Madison, O. Karampela, F. Ullén, L. Holm, Effects of practice on variability in an isochronous serial interval production task: asymptotical levels of tapping variability after training are similar to those of musicians, Acta Psychol (Amst), 143 (2013), 119-128.

[9] J.A. Seitz, Dalcroze, the body, movement and musicality, Psychol. Music 33 (2005), 419-435.

\section{Mr. Keita Ueda}

He graduated master course at Graduate School of Engineering in Kyoto University and received Dr. (Eng.) from Kyoto University. $\mathrm{He}$ is now a member of Graduate School of Education in Hiroshima University. He is a member of IEEE, SICE, and ISCIE.

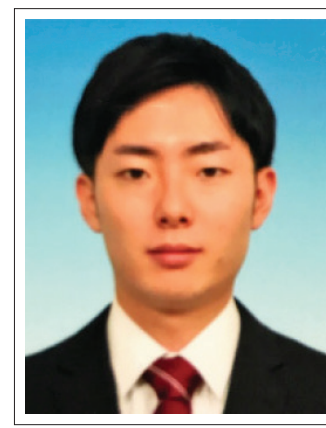

He graduated Course of Technology and Information Education in School of Education in Hiroshima University. 